\section{Putting the neo into neoangiogenesis}

\author{
Malcolm A.S. Moore
}

\section{Commentary}

See related article, pages $337-346$.

Memorial Sloan-Kettering Cancer Center, 1275 York Avenue,

New York, New York 10021, USA.

Phone: (212) 639-7090; Fax: (212) 717-3618; E-mail: m-moore@ski.mskcc.org.

J. Clin. Invest. 109:313-315 (2002). DOI:10.1172/JCI200214940.

Angiogenesis is the process by which new blood vessels develop by sprouting of preexisting vessels, and until recently this was considered the sole mechanism of blood vessel formation in postnatal life. The development of the vascular system in the embryo, termed vasculogenesis, is due to in situ differentiation of endothelial progenitors or angioblasts. Studies over the last five years have modified this dogma, since endothelial stem cells have been identified in the adult and have been shown to participate in new blood vessel formation in normal and pathological states, including tumor "neoangiogenesis" (1-5).

\section{Defining the adult and embryonic angioblast}

The importance of the bone marrow as a source of endothelial stem/progenitor cells was first demonstrated in a canine bone marrow transplantation model, in which the marrow cells from the donor and recipient were genetically distinct (1). Dacron grafts implanted in the descending thoracic aorta develop endothelial linings that are of donor marrow origin. A rare cell - found at a frequency of about 1:10,000 nucleated cells in human marrow, in G-CSF-mobilized peripheral blood, and in umbilical cord blood - expresses the receptor for VEGF (VEGFR2/Flk1/KDR), which is absent on hematopoietic stem cells (HSCs) $(1,2)$. These cells share with HSCs the expression of CD34 and CD133, the latter being absent on mature endothelium. In the presence of endothelial growth medium containing VEGF, these $\mathrm{CD} 133^{+} \mathrm{KDR}^{+}$ cells form late-developing colonies of endothelium and are considered to be endothelial progenitors or angioblasts (2). Recently, mutations in the Id gene have been shown to impair tumor angiogenesis in mice; transplantation of wild-type marrow or VEGF-mobi- lized angioblasts into such mice restores their capacity to support rapid tumor growth with donor neovascular development (3).

Developmentally, Flk1 ${ }^{+}$cells that can differentiate into either hematopoietic cells or endothelium (hemangioblasts) appear in the para-aortic splanchnopleura, the yolk sac blood islands, and subsequently the aorta-gonad-mesonephros (AGM) region of the developing embryo (Figure 1a) (6). Embryonic stem cell-derived embryoid bodies con- tain a unique precursor population that, in response to VEGF, gives rise to blast colonies in semisolid medium (7). These blast colonies are equivalent to the hemangioblast, and, upon transfer to liquid culture with appropriate cytokines, they generate both hematopoietic and endothelial cells (Figure 1a) $(7,8)$. Despite extensive efforts to demonstrate the hematopoietic potential of adult $\mathrm{CD} 34^{+} \mathrm{CD} 133^{+} \mathrm{KDR}^{+}$cells, there has been no clear evidence that cells of this phenotype are comparable

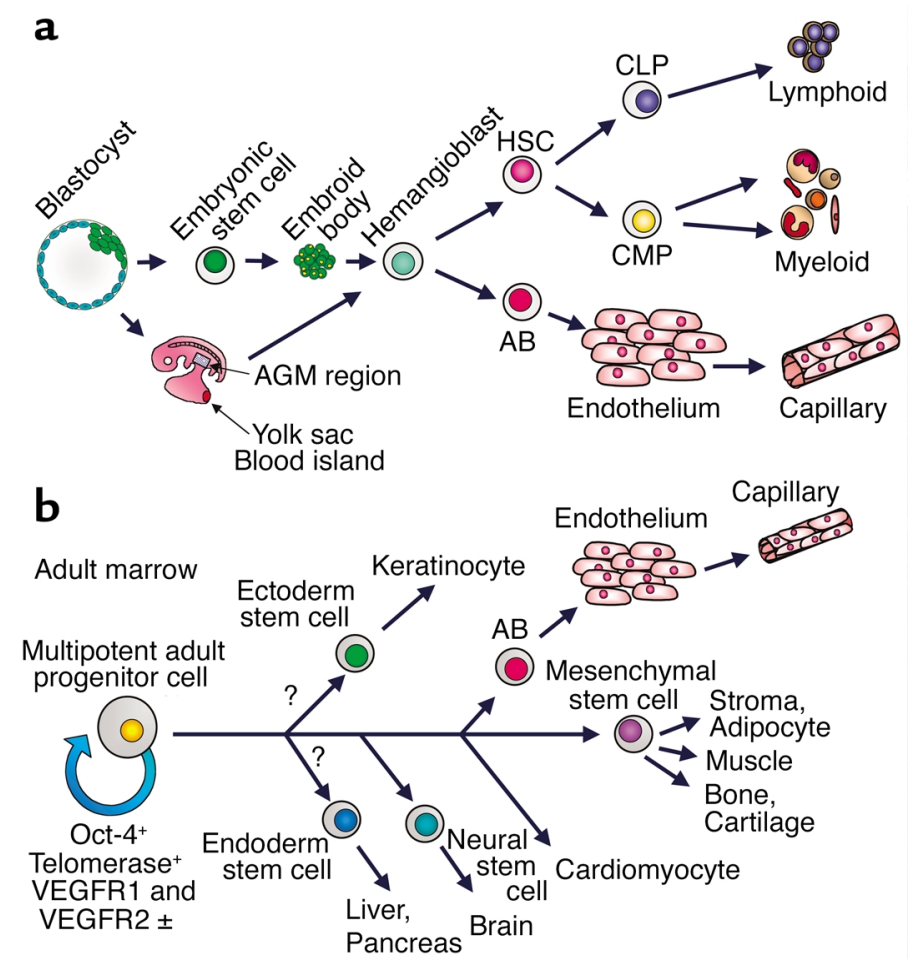

Figure 1

(a) Generation of hemangioblasts from blastocyst-derived embryonic stem (ES) cells, or from the yolk sac or AGM region of the early embryo. Upon appropriate cytokine stimulation, hemangioblasts differentiate into angioblasts $(A B)$ that differentiate into endothelium, which can then form capillaries. Hemangioblasts also generate HSCs, which in turn generate common lymphocyte progenitors (CLP) that differentiate into T, $\mathrm{B}$, and NK cells, and common myeloid progenitors (CMP) that differentiate to erythroid, granulocyte-macrophage and megakaryocytic progenitors. (b) The pathway of generation of angioblasts and endothelial cells from the adult marrow MAPCs based on published data (16-18) showing endothelial differentiation as well as generation of MSCs and their differentiated progeny, and neural differentiation. Question marks indicate possible, not yet documented, differentiation pathways that could generate ectodermal and endodermal tissues. \pm , weekly positive. 
to the hemangioblasts identified in embryonic stem (ES) cell differentiation models and in studies of the early embryo.

\section{Stem cells and developmental plasticity}

Stem cells have been identified in most organ tissues, including hematopoietic, neural, gastrointestinal, epidermal, and hepatic. Mesenchymal stem cells (MSCs), first identified in the bone marrow as fibroblast colony-forming cells, have been subsequently shown to differentiate into bone, cartilage, adipocytes, hematopoietic support stroma, and skeletal myocytes (9). On the basis of both phenotypic analysis and transplantation studies, MSCs are readily distinguished from HSCs. The bone marrow has also been shown to contain cells that can differentiate into neural elements, hepatocytes, cardiomyocytes, and epithelial cells of the lung, gastrointestinal tract, and skin, leading to an explosive growth of studies suggesting stem cell plasticity (10-13).

The term "plasticity" is best used to indicate that cells that have undergone lineage-specific differentiation can, in some way, transdifferentiate, or dedifferentiate to a more primitive stage and be reprogrammed to differentiate into a quite different pathway. As reported, this reprogramming can cross germ lines, as, for example, neural cells of neurectodermal origin developing from mesoderm-derived HSCs and vice versa (10). An alternative view is that different types of stem cells coexist in different sites. According to this concept, the reason that cells in the subventricular region of the brain, or isolated from striated muscle, can reconstitute the hematopoietic system is that HSCs are ubiquitous and copurify with populations isolated on the basis of common markers (CD34, CD133, c-kit) or that share a distinctive profile of Hoechst dye staining (called the side population) $(14,15)$.

In addition, some adult tissues, such as the marrow, may also contain rare cells that are more multipotent than any of the lineage-specific stem cells. Such cells, it used to be assumed, must be restricted to very early stages of development, similar perhaps to ES cells or primordial germ cells. While this assumption is now in question, doubts continue to plague the field about the rigor of claims that disparate tissues share common clonal origins. Cell sorting, single-cell deposition techniques, and limiting dilution cultures and transplants have generally been used in lineage studies. The alternative approach of following lineages marked with unique retroviral integration sites has not yet been widely pursued.

\section{Multipotent adult progenitor cells}

To add further complexity to the stem cell debate, Catherine M. Verfaillie and her associates have identified a unique cell in human and rodent postnatal marrow that they term the multipotent adult progenitor cell (MAPC) (16-18). MAPCs were selected by depleting adult bone marrow of hematopoietic cells expressing CD45 and glycophorin-A, followed by long-term culture on fibronectin with EGF and PDGF under low serum conditions. Under these conditions, a cell population emerges that does not undergo proliferative senescence, since it expresses telomerase and has long telomeres that do not shorten over 80 population doublings (PD). These cells are, nevertheless, cytogenetically stable. Their surface markers define a unique phenotype that distinguishes them from any other known class of stem cells. They lack such stem cell markers as CD34 and c-kit, as well as HLA-DR and class I HLA, but they express AC133 and low levels of VEGFR2/Flk1/KDR, as well as VEGFR1/Flt1 and the ES marker Oct-4. Retroviral marking shows definitively that a single MAPC can differentiate to both an MSC that produces cells of limb-bud mesoderm derivation (osteoblasts, chondrocytes, adipocytes, stromal cells, and skeletal myoblasts), and visceral mesoderm that differentiates into endothelium (Figure 1b) (16).

The study of Reyes et al. reported in this issue of JCI evaluates in depth the potential of human MAPCs, obtained after 20-65 PD, to form functional endothelium (17). Culture of the cells with VEGF induces differentiation into $\mathrm{CD}^{4} 4^{+}$, vascular endothelial-cadherin ${ }^{+}$, Flk1/KDR ${ }^{+}$cells, a phenotype consistent with angioblasts (Figure 1b). Subsequently the cells express $\mathrm{vWF}$ and markers of mature endothelium, such as CD31, CD36, and CD62-P. This mature endothelium can be expanded further ( 20 PD), but it lacks the apparent immortality of the MAPC. The study evaluated the function of MAPC-generated endothelium, showing that the cells form vascular tubes when plated on Matrigel and upregulate angiogenic receptors and VEGF in response to hypoxia. In immunoincompetent mice, intravenously injected endothelial cells contribute to neovascularization of transplanted tumors and can participate in wound healing. Remarkably, even injection of undifferentiated MAPC results in human endothelial cells vascularizing a spontaneous murine tumor, indicating that undifferentiated MAPC can respond to local differentiation-inducing cues in vivo. Despite extensive efforts, hematopoietic differentiation of human MAPC has not been observed, indicating a significant difference from the pathway by which both hematopoietic and endothelial cells are generated from hemangioblasts. ES cells, in contrast, are easily differentiated into hematopoietic as well as endothelial elements (Figure 1).

\section{MAPCs and ES cells}

Undoubtedly the recognition of MAPCs will alter the debate regarding the need for ES cells or therapeutic cloning in order to generate tissues for organ repair. The major problem for adult stem cells, as previously understood, was that their proliferative potential would be limited by the age of the donor, with progressive telomere shortening with each cell division leading to senescence after 20-30 PD. In addition, extensive stem cell expansion without differentiation was not achievable with most types of adult stem cell, the HSC being the most extensively investigated in this regard. MAPC lines appear to share with ES lines an extensive if not infinite proliferative potential with cytogenetic and telomeric stability and retention of multipotentiality. It remains to be seen whether the MAPC lines accumulate the genetic damage of their donors an issue of concern if they are harvested for therapeutic reasons from the bone marrow of elderly individuals who may have had prior chemo- or radiotherapy. While ES cells can be induced to differentiate into at least 100 different cell and tissue types in vitro and can generate all the lineages that constitute the adult organism, the range of differentiation reported for MAPC comprises the mesodermal tis- 
sues described in published reports, including cardiac as well as smooth and skeletal muscle $(16,17)$. In addition, Verfaillie and colleagues have evidence that MAPCs can be induced to differentiate to cells of the neurectodermal lineage, including $\beta$ tubulinIII-, neurofilament-, neuron-specific enolase-, and glutamate-positive neurons, glial fibrillar acidic protein-positive astrocytes, and myelin basic protein- and galactocerebroside-positive oligodendrocytes (18). The neural (and potentially other) pathways of differentiation have not yet been shown to share a common origin with the cells generating mesodermal lineages, since a minimum of ten cells is required for passage of MAPCs. However, the retention of multiple lineage differentiation potential for more than $80 \mathrm{PD}$ strongly supports the common origin for mesodermal and neurectodermal tissues from a MAPC.

The clinical applications of MAPCs are extensive, even if we restrict our consideration to the current demonstration of generation of potentially vast numbers of functionally normal endothelial cells with extensive proliferative potential. A therapeutic role of MAPC-derived angioblasts/endothelium is indicated in treatment of myocardial infarction. Angioblasts from human marrow can directly induce new blood vessel formation in sites of myocardial infarction, resulting in decreased apoptosis of hypertrophied myocytes in the peri-infarct region, long-term salvage and survival of viable myocardium, and sustained improvement in cardiac function (5).

Telomeric shortening and proliferative senescence of endothelium occurs in coronary arteries and other sites of high hemodynamic stress, and endothelial replacement therapy may be considered here. Endothelial cells may also be used to facilitate wound healing and have potential as targets for gene delivery for correction of genetic disease. Injection of endothelial cells that can home to and integrate into the vasculature of developing tumors may provide a selective antitumor drug delivery system. Unlike ES cell-derived endothelium, the MAPC-derived cells could be autologous, since the data presented suggest that while the MAPCs are extremely rare ( 1 in $10^{7}$ to 1 in $10^{8}$ marrow cells), lines have been reproducibly generated from the marrow of many donors over a wide age range (refs. 16-18). Ultimately, an extensive comparison of MAPC with ES cells and cells generated by therapeutic cloning will be necessary before a final decision can be made concerning the respective merits of these cell sources for generation of tissues for repair of damaged or degenerating tissues and organs.

1. Shi, Q., et al. 1998. Evidence for circulating bonemarrow-derived endothelial cells. Blood. 92:362-367.

2. Peichev, M., et al. 2000. Expression of VEGFR-2 and $\mathrm{AC} 133$ by circulating human $\mathrm{CD} 34^{+}$cells identifies a population of functional endothelial precursors. Blood. 95:952-958.
3. Lyden, D., et al. 2001. Impaired recruitment of bone-marrow-derived endothelial and hematopoietic precursor cells blocks tumor angiogenesis and growth. Nat. Med. 7:1194-1201.

4. Jackson, K.A., et al. 2001. Regeneration of ischemic cardiac muscle and vascular endothelium by adult stem cells. J. Clin. Invest. 107:1395-1402.

5. Kocher, A.A., et al. 2001. Neovascularization of ischemic myocardium by human bone-marrowderived angioblasts prevents cardiomyocyte apoptosis, reduces remodeling and improves cardiac function. Nat. Med. 7:430-436.

6. Lacaud, G., et al. 2001. Regulation of hemangioblast development. Ann. NY. Acad. Sci. 938:96-108.

7. Choi, K., et al. 1999. A common precursor for hematopoietic and endothelial cells. Development. 125:725-732.

8. Hirashima, M., et al. 1999. Maturation of embryonic stem cells into endothelial cells in an in vitro model of vasculogenesis. Blood. 93:1253-1263.

9. Deans, R.J., and Moseley, A.B. 2000. Mesenchymal stem cells: biology and potential clinical uses. Exp. Hematol. 28:875-884.

10. Moore, M.A.S. 1999. "Turning brain into blood": clinical applications of stem-cell research in neurobiology and hematology. N. Engl. J. Med. 1341:605-607.

11. Lagasse, E., et al. 2000. Purified hematopoietic stem cells can differentiate into hepatocytes in vivo. Nat. Med. 6:1229-1234.

12. Orlic, D., et al. 2001. Bone marrow cells regenerate infarcted myocardium. Nature. 410:701-705.

13. Krause, D.S., et al. 2001. Multi-organ, multi-lineage engraftment by a single bone-marrow-derived stem cell. Cell. 105:369-377.

14. Jackson, K.A., Mi, T., and Goodell, M.A. 1999. Hematopoietic potential of stem cells isolated from murine skeletal muscle. Proc. Natl. Acad. Sci. USA. 96:14482-14486.

15. Kawada, H., and Ogawa, M. 2001. Bone marrow origin of hematopoietic progenitors and stem cells in murine muscle. Blood. 98:2008-2013.

16. Reyes, M., et al. 2001. Purification and ex vivo expansion of postnatal human marrow mesodermal progenitor cells. Blood. 98:2615-2625.

17. Reyes, M., et al. 2002. Origin of endothelial progenitors in human postnatal bone marrow. J. Clin. Invest. 109:337-346. DOI:10.1172/JCI200214327.

18. Reyes, M., and Verfaillie, C.M. 2001. Characterization of multipotent adult progenitor cells, a subpopulation of mesenchymal stem cells. Ann. NY. Acad. Sci. 938:231-235. 